\begin{tabular}{|l|c|c|c|}
\hline ACTA CLASSICA & LII. & 2016. & \\
UNIV. SCIENT. DEBRECEN. & & & pp. 135-147. \\
\hline
\end{tabular}

\title{
DAS BILDPROGRAMM DER MELEAGER-PLATTE AUS DEM SEUSO-SCHATZ
}

\author{
VON TAMÁS GESZTELYI
}

\begin{abstract}
There are eight distinct love stories on the Meleager plate of the Seuso treasure. In four of them hunting plays an important role. The main theme of the depicted stories is allprevailing love, the emotion that is the motivating force in human life. An ancillary theme of the stories is hunting, which can be interpreted in a concrete as well as a figurative sense. Hunting is an aristocratic pleasure but at the same time it represents the exercise of virtues, too, in which even females can participate, cf. the scenes with Helene and Atalante. Although there is no need to look for a topical event to feature the motif of love, it is tempting to determine the contemporary function of the treasure as a wedding gift. We are inclined to believe that Seuso, the well-off owner of the treasure, must have been given this unique silver tableware as a wedding gift some time around the beginning of the 5th century.

Keywords: Seuso treasure, Meleager plate, iconographic program, love-stories.
\end{abstract}

Der Seuso-Schatz ist eines der bedeutendsten Denkmäler der spätantiken Goldschmiedekunst. Er enthält das schönste festliche Tafelsilber, das aus dieser Zeit erhalten geblieben ist. Es handelt sich um insgesamt 14 Gefäße: 4 Platten, 5 Kannen, ein Toilette-Kästchen, 2 Eimer, eine Waschschale und eine Amphore. ${ }^{1}$ Des Weiteren wird sein Wert für uns noch dadurch gesteigert, dass er möglicherweise ursprünglich aus Pannonien stammt ${ }^{2}$ und zum Teil auch nach Ungarn zurückgeholt wurde, was allerdings nicht für die Meleager-Platte gilt. Die einzelnen Gefäße sind in verschiedenen Techniken verziert worden, zum Teil geometrisch, zum Teil figurativ. Das lässt darauf schließen, dass sie aus verschiedenen Werkstätten stammen, also nicht von vorneherein zusammengehörten, sondern durch die Sammlungs- bzw. Kauftätigkeit des Besitzers zu einem Tafelsilber vereinigt wurden.

\footnotetext{
${ }^{1}$ Mango - Bennett 1994.

${ }^{2}$ Visy 2012.

doi $10.22315 / \mathrm{ACD} / 2016 / 10$
} 
Das figurativ am reichsten verzierte Stück des Ensembles, die MeleagerPlatte, wurde nach der in ihrem Zentrum zu sehenden Meleager-Szene benannt $(A b b .9)$. Auf ihrem Rand verläuft ein in sechs Segmente gegliedertes Band von mythologischen Szenen, im Mittelpunkt der Platte befindet sich die siebente, namensgebende Szene. ${ }^{3}$ Mythologische Szenen verzieren auch die AchillesPlatte, auf deren Rand die abgebildeten Geschichten in vier Einheiten gegliedert sind und in deren Mittelpunkt die Geschichte von 'Achilles auf Skyros' zu sehen ist. ${ }^{4}$ Mythologische Szenen verzieren noch zwei Eimer und die Wand einer Kanne. Das Thema aller drei Darstellungen ist dasselbe: der Augenblick der Trennung von Hippolytos und Phaidra. ${ }^{5}$ Mit Recht kann man fragen, ob bei der Zusammenstellung dieser mythologischen Geschichten der Zufall die Hauptrolle gespielt haben mag oder ob sie bewusst ausgewählt wurden und wenn ja, welche Absicht dahinter gestanden haben mag.

In Bezug auf die Dichter und Schriftsteller wird diese Frage in mehreren Fällen klar beantwortet. Horaz formulierte die Antwort in seiner Ars poetica folgendermaßen: Aut prodesse volunt aut delectare poetae, aut simul et iucunda et idonea dicere vitae (Ep. II 3, 353-4). Dichtung kann also nützlich sein, sie kann unterhaltsam sein und sie kann beides miteinander verbinden. Plinius d. Ä. stellt den Nutzen in den Vordergrund. Alles, was er geschrieben hatte, wollte er dem römischen Volk zur Verfügung stellen: populo Romano praestitisse (Nat. Praef. 16) bzw. allen Sterblichen damit helfen: iuvare mortalem (Nat. II 18). Macrobius führt im Vorwort zu den Saturnalien aus, dass gute Literatur unterhält, bildet und nützlich ist: in quibus ... invenies plurima quaesit aut voluptati legere aut cultui legisse aut usui meminisse (Praef. 10).

In der bildenden Kunst war es wohl nicht anders. Tafelsilber war zweifellos in erster Linie nützlich, wollte zugleich aber auch Entzücken hervorrufen. Da aber mythologische Geschichten zusammengestellt wurden, stellt sich die Frage nach der Absicht, ob nämlich durch die Geschichten mit Hilfe positiver oder negativer exempla Bildung vermittelt wird. Erziehung findet nämlich mit Hilfe von Beispielen statt, wie Macrobius meint (Sat. Praef. 1).

Widmen wir uns zunächst den auf der Meleager-Platte abgebildeten Geschichten! Von unten und von links nach rechts fortschreitend ist im ersten Segment das Urteil des Paris zu sehen (Abb. 1). ${ }^{6}$ Paris sitzt am linken Rand, seine linke Hand hält er auf seiner auf den Schenkel gestützten syrinx. Rechts von ihm steht Hermes, den Kopf wendet er Paris zu, als warte er auf dessen Entscheidung, in der ausgestreckten Rechten hält er den der Siegerin zustehen-

\footnotetext{
${ }^{3}$ Mango - Bennett 1994, 98-152.

${ }^{4}$ Mango - Bennett 1994, 153-180.

${ }^{5}$ Mango - Bennett 1994, 319-401

${ }^{6}$ Mango - Bennett 1994, 125-128.
} 
den goldenen Apfel. An der Spitze der Göttinnen steht Aphrodite, womit signalisiert wird, dass sie den Apfel bekommen wird. Hinter ihr steht die voll bewaffnete Athene, als dritte folgt auf ihrem Thron sitzend Hera, die ihre Rechte ausstreckt, als ob sie hoffen würde, den Apfel zu bekommen. Am rechten Rand stützt sich eine Frauengestalt auf eine Säule, wahrscheinlich Helena, die Belohnung des verliebten Paris.

In der griechischen Kunst findet sich vor allem das erste Motiv der in der bildenden Kunst äußerst beliebten Geschichte: Hermes führt die Göttinnen vor Paris. ${ }^{7}$ Auf römischen Darstellungen ist das Motiv der Vorbereitung des Urteils am häufigsten zu finden: Hermes reicht Paris den goldenen Apfel, damit dieser den Wettbewerb entscheide, während er selbst im Hintergrund verbleibt. ${ }^{8}$ Die Besonderheit der Ikonographie der Meleager-Platte besteht darin, dass Hermes den Apfel nicht Paris, sondern der siegreichen Aphrodite reicht, aber offensichtlich auf Grund der Entscheidung des Paris. Einzig eine pyxis aus Elfenbein aus dem 6. Jahrhundert stellt die Szene so dar, dass Hermes Aphrodite den goldenen Apfel reicht, während Paris gar nicht zu sehen ist. ${ }^{9}$ Auf der frühbyzantinischen pyxis dienten Auswahl und Darstellung der Geschichte offensichtlich dem Ziel, Aphrodite als Verkörperung der weiblichen Schönheit zu zeigen. Es ist überraschend, wie ungeschminkt dies in offensichtlich bereits christlicher Umgebung geschieht. Eine weitere Eigenart der Meleager-Platte besteht darin, dass auch Helena anwesend ist, womit die Fortsetzung des Liebesstrangs der Geschichte betont wird.

Das zweite Segment stellt den Perseus-Zyklus dar (Abb. 2). ${ }^{10}$ Am linken Rand sieht man den mit gezücktem Schwert laufenden Akrisios, der seine Tochter mit dem kleinen Perseus auf dem Arm von seinem Hof vertreibt. In der nächsten Szene befreit der bereits erwachsene Perseus Andromeda von dem ihr Leben bedrohenden Seeungeheuer und bringt so das Haupt der Gorgo in seinen Besitz. Rechts von ihnen ist der Fischer Diktys zu sehen, der die mit ihrem Kind ans Ufer gespülte Danaë gefunden hatte. Schließlich sitzt wahrscheinlich die männliche Personifikation der Insel Seriphos mit einem großen Füllhorn auf einem Felsen. Das ikonographisch einzigartige Element des Zyklus ist die Szene der Vertreibung. Akrisios jagt seine Tochter nie mit gezücktem Schwert. ${ }^{11}$ Wenn er überhaupt auf griechischen Vasenbildern erscheint, dann als alter Mann, der resigniert neben der Truhe steht, die Danaë und Perseus für ihn ge-

\footnotetext{
${ }^{7}$ Kossatz-Deissmann 1994, Nr. 1-60.

${ }^{8}$ Kossatz-Deissmann 1994, Nr. 71-82.

${ }^{9}$ Kossatz-Deissmann 1994, Nr. 102, p. 188.

${ }^{10}$ Mango - Bennett 1994, 129-132.

${ }^{11}$ Maffre 1986 Nr. 41-69.
} 
fertigt hatten. ${ }^{12}$ Andromedas Rettung folgt der konventionellen Ikonographie, wie wir sie auch auf einem Wandgemälde in Pompeji sehen können: Nachdem Perseus den Ketos getötet hat, dessen Leib in der linken Ecke liegt, hilft er mit seiner Rechten Andromeda beim Abstieg vom Felsen, in seiner Linken hält er seine Waffe, die Harpe und das abgeschlagene Gorgonenhaupt. ${ }^{13}$ Die zwei letzten Gestalten, der Fischer (Diktys) und die Personifikation der Insel, sind dagegen keine organischen Bestandteile der konventionellen Ikonographie.

Das dritte Segment stellt die Geschichte von Hippolytos und Phaidra dar (Abb. 3). ${ }^{14}$ Am linken Rand sitzt Phaidra auf ihrem Thron, vor ihr die Amme, welche Hippolytos die Liebe ihrer Herrin mitzuteilen versucht. Der zu Pferd erscheinende Hippolytos weist diese zurück, wirft Phaidras Liebesbrief auf den Boden und reitet in Richtung seines Jagdgefährten, der mit zwei Jagdhunden auf ihn wartet. Am rechten Rand des Bildes ist wieder eine Gestalt $\mathrm{zu}$ sehen, die ein Füllhorn trägt, diesmal eine Frauengestalt mit einer Mauerkrone auf dem Kopf, folglich eine Stadtgöttin, wahrscheinlich die Tyche des Schauplatzes der Ereignisse, Athens. Die Darstellung der tragisch ausgehenden Trennung von Hippolytos und Phaidra ist ein Produkt der römischen Kunst. Auf griechischen Vasenbildern begegnen wir Hippolytos nur auf seinem Gespann, dessen Pferde vor dem sich aus dem Meer erhebenden Stier erschreckend durchgehen. ${ }^{15}$ Der Liebesbrief, den die Amme Hippolytos übergibt, ist ebenfalls ein Motiv römischen Ursprungs, in der von Euripides bekannten Geschichte kommt es nicht vor, dafür umso häufiger auf römischen Wandgemälden und Reliefs, aber auch dort nicht immer. ${ }^{16}$ Die Tyche Athens ist als individuelles Motiv der Meleager-Platte zu betrachten. Die Popularität der Geschichte auf dem Tafelsilber wird durch das weitere Vorkommen auf zwei Eimern und einer der Kannen unterstrichen (Abb. 4). Sie sind ikonographisch identisch. ${ }^{17}$ Die Geschichte wird mit Hilfe eines Gebäudeeingangs in zwei Teile gegliedert: links sitzt die von ihren Bediensteten umgebene, trauernde Phaidra, rechts steht der von seinen Jagdgefährten umgebene, aufbruchsbereite Hippolytos, daneben kniet die flehende Amme. Die kniende Körperhaltung kommt nur auf einem pannonischen und einem norischen Relief vor. ${ }^{18}$

\footnotetext{
${ }^{12}$ Maffre 1981, Nr. 1-11.

${ }^{13}$ Schauenburg 1986, Nr. 67-89; Roccos 1994, Nr. 203-216.

${ }^{14}$ Mango - Bennett 1994, 133-135.

${ }^{15}$ Linant de Bellefonds 1990, 459.

${ }^{16}$ Linant de Bellefonds 1990, 460.

${ }^{17}$ Mango - Bennett 1994, 389-396.

${ }^{18}$ Gesztelyi 2015, 69. f.
} 
Die meisten ikonographischen Neuerungen enthält das vierte Segment, auf dem die Geschichte von Pyramus und Thisbe dargestellt wird (Abb. 5). ${ }^{19} \mathrm{Am}$ linken Rand sitzt mit trauernder Körperhaltung eine Wassernymphe, die sich mit ihrer Rechten auf einen umgestürzten Krug stützt, aus dem Wasser rinnt. Sie wird gefolgt vom nach seinem Selbstmord auf dem Boden liegenden Pyramus, dessen Linke leblos unter seine Körper verdreht ist und dessen Schwert auf dem Boden liegt. Rechts von ihm ist eine weitere Wassernymphe zu sehen, die sich ebenfalls auf einen umgestürzten Krug stützt und in der anderen Hand eine Wasserpflanze hält. Oberhalb von ihnen fasst ein Panther mit Pfote und Maul einen Umhang oder ein Tuch. Rechts ist die verzweifelt vom Schauplatz fliehende Thisbe zu sehen, vor ihr ein umgestürzter Krug, aus dem Wasser rinnt. Die Szenenfolge wird von einem auf einer Syrinx spielenden Mann, vielleicht einem Hirten, abgeschlossen.

Wir sehen zweifellos die von Ovid ${ }^{20}$ bekannte Geschichte von Pyramos und Thisbe, die ihren Weg auch in die bildende Kunst gefunden hat. Mehrere Wandgemälde in Pompeji folgen treu der Beschreibung Ovids. ${ }^{21}$ Die Begegnung fand am Grab von Ninos statt - dieses sehen wir auf dem einen Wandgemälde auf der linken Seite -, und zwar unter einem Maulbeerbaum - diesen sehen wir auf der rechten Seite. Auf dem anderen Wandgemälde erscheint im Hintergrund der sich entfernende Löwe, der Umhang ist an dem Baum hängengeblieben. Im Vordergrund wird der Ausgang der Geschichte dargestellt: Pyramos liegt bereits tot am Boden, Thisbe tötet sich mit seinem Schwert ebenfalls selbst. Die Metamorphose findet auf dem Maulbeerbaum statt, dessen weiße Früchte durch das verströmte Blut dunkelrot werden.

Auf der Meleager-Platte sehen wir nicht genau diese Geschichte. Erstens fehlen die bestimmenden Elemente des Ovidschen Schauplatzes, das Grab des Ninos und der Maulbeerbaum, zweitens erscheinen neue Figuren wie die Wassernymphen, drittens folgt Thisbe ihrem Liebhaber nicht in den Tod, sondern flieht vom Schauplatz. Die Abweichungen können damit erklärt werden, dass es eine zweite, kilikische Version der Geschichte gab. Von ihr wissen wir allerdings nur durch spätantike Anspielungen. Nonnos schreibt in seinem großen Epos, den Dionysiaca, dass Pyramos Thisbe so suche wie Alpheios Arethusa während der nach dem Tod des Zagreus einsetzenden Flut. ${ }^{22}$ Dass dahinter die Geschichte einer Metamorphose steht, ergibt sich aus einer anderen Textstelle bei Nonnos, wo im Zusammenhang mit verschiedenen Metamorphosen auch

\footnotetext{
${ }^{19}$ Mango - Bennett 1994, 136-139.

${ }^{20}$ Met. 4, 55-166.

${ }^{21}$ Linant de Bellefonds 1994 Nr. 19-22.

${ }^{22}$ Dion. VI 344 ff.; Türk 1937, 286; Knox 1989, 322.
} 
die Namen von Pyramos und Thisbe zu finden sind. ${ }^{23}$ Für den kilikischen Ursprung der Geschichte spricht, dass es dort einen Fluss namens Pyramos gab. ${ }^{24}$ Wahrscheinlich erzählte die Geschichte von Pyramos und Thisbe vom Ursprung der Benennung. Auf der Meleager-Platte sind die zwei Pyramos in die Mitte nehmenden Wassernymphen als Anspielung auf seine Metamorphose in den Fluss zu verstehen, Thisbes Verwandlung in die Quelle bringt wiederum das aus dem zu ihren Füßen liegenden Krug fließende Wasser zum Ausdruck.

Auch auf Denkmälern der bildenden Kunst in den östlichen Gebieten finden wir die Spur der Darstellung von Pyramos und Thisbe als Wassergötter. Auf einem antiochischen Mosaik aus der Zeit der Severer erscheint auf je einem Medaillon das Brustbild der beiden Gestalten auf ihrem Boot mit Wasserpflanzen. $^{25}$ Die Identifizierung der Gestalten ist durch die Aufschriften eindeutig möglich. Auf Basis der kilikischen Version des Mythos dürfte auch um die Wende vom 3. zum 4. Jahrhundert n. Chr. in Nea Paphos das Mosaik entstanden sein, auf dem rechts Pyramos als Flussgott auf dem Boden liegend dargestellt ist, während er sich auf einen zur Seite geneigten Krug stützt, aus dem Wasser fließt (Abb. 6). In seiner linken Hand hält er eine Wasserpflanze, in der rechten ein Füllhorn. Links ist die fliehende Thisbe zu sehen, in der Mitte im Hintergrund ein Panther, der am Umhang zerrt. ${ }^{26}$ Das Mosaik zeigt uns also die Metamorphose des Pyramos als vollendete Tatsache. Was mit Thisbe passiert, sehen wir aber nicht. Nichts deutet darauf hin, dass sie sich selbst töten würde. Allerdings gibt es einen spätantiken Rhetor namens Nikolaos, der Thisbes Selbstmord erwähnt, den sie deswegen begangen habe, weil sie schwanger gewesen sei, Pyramos wiederum sei ihr in den Tod gefolgt. ${ }^{27}$ Aus Mitleid hätten die Götter Pyramos in einen Fluss und Thisbe in eine Quelle verwandelt, die nebeneinander ins Meer flössen. Diese Version ist aber mit großer Wahrscheinlichkeit in der rhetorischen Tradition entstanden ${ }^{28}$, mit der die beiden erhalten gebliebenen Darstellungen nicht übereinstimmen, da auf ihnen Tod und Metamorphose des Pyramos vorher stattfinden und an der fliehenden Thisbe keine Spuren der Schwangerschaft zu erkennen sind. Aus der Darstellung auf der Meleager-Platte können wir eher darauf schließen, dass Thisbe sich aus Schmerz in eine Quelle verwandelt hat.

Festes Element der Darstellungen der Geschichte in der Ovidschen Tradition ist der Löwe, in der kilikischen der Panther, der Thisbes Umhang mit seinem

\footnotetext{
${ }^{23}$ Dion. XII 84; Türk 1937, 286.

${ }^{24}$ Knox 1989, 326 f.

${ }^{25}$ Linant de Bellefonds 1994 Nr. 1; Linant de Bellefonds 1994a, 346.

${ }^{26}$ Linant de Bellefonds 1994 Nr. 24.

${ }^{27}$ Türk 1937, 287.

${ }^{28}$ Knox 1989, 324.
} 
blutigen Maul zerreißt. Daraus können wir schließen, dass dieses in beiden Traditionen wichtige Motiv Teil der ursprünglichen Geschichte gewesen ist. Ob sie tatsächlich babylonischen Ursprungs ist und von dort nach Kilikien gelangte oder ob Ovid sie dorthin verlegte, wäre schwer zu entscheiden. Es kann aber festgestellt werden, dass die kilikische Version des Mythos am vollständigsten in der Darstellung auf der Meleager-Platte erhalten geblieben ist, welche mit dem aus dem Krug fließenden Wasser auch eine Anspielung auf die Metamorphose der Thisbe enthält.

Das fünfte Segment zeigt eine nicht genau zu bestimmende Geschichte ohne Parallele. Im Mittelpunkt steht ein Mann mit Schild und Lanze, mit einer phrygischen Mütze auf dem Kopf, der den linken Fuß auf den Rücken eines erlegten Löwen setzt $(A b b$. 7). Hinter ihm steht eine sich auf ihre Lanze stützende Frauenfigur, die ihre rechte Hand auf die Schulter des Mannes legt. Nach MangoBennett ${ }^{29}$ handle es sich um Paris und Helena, obwohl es kein Beispiel für die Darstellung von Paris als Jäger gibt und noch weniger von Helena. Als Hirte konnte Paris natürlich in eine Situation geraten, in der er einen seine Herde angreifenden Löwen töten musste. Die für ihn typische Waffe war aber nicht die Lanze, sondern der Bogen. Die Szene wird von zwei jungen Reitern gesäumt, sicherlich die als berühmte Jäger bekannten Dioskuren, Helenas Geschwister. Am rechten Rand des Bildes ist ein Flussgott zu sehen, nach MangoBennett sicherlich Eurotas, was auf Lakonien als Schauplatz hindeuten würde.

Im sechsten Segment steht sich ebenfalls ein Jägerpaar gegenüber $(A b b .8)$. Rechts Meleager, der in seiner Rechten ein abgezogenes Eberfell hält, das er der vor ihm stehenden Atalante entgegenstreckt. ${ }^{30}$ Atalante hält in der Linken einen Bogen, in der Rechten eine große Opferplatte. Die Szene ist ikonographisch ohne Parallele. Meleager erscheint in Darstellungen üblicherweise sitzend, wie wir es auch auf einem Wandgemälde in Pompeji bzw. auf dem im Mittelpunkt stehenden Medaillon sehen könne. ${ }^{31}$ Auf kaiserzeitlichen Mosaiken kommen die beiden nebeneinanderstehend mit dem erlegten Eber zwischen ihnen auf dem Boden vor. ${ }^{32}$ Auf der Meleager-Platte ist aber nicht mehr das Tier, sondern nur noch sein abgezogenes Fell zu sehen, welches der Held Atalante anbietet. Atalante hält die Platte in ihrer Rechten wohl zum Zweck des Dankopfers hoch.

Die zentrale Szene wird von je einer weiteren Darstellung einer Liebesgeschichte gesäumt. Rechts stehen sich Aphrodite und Adonis mit einem Jagdhund gegenüber, links ist Leda mit dem Schwan und einem kleinen Eros von

\footnotetext{
${ }^{29}$ Mango - Bennett 1994, $140 \mathrm{ff}$.

${ }^{30}$ Mango - Bennett 1994, 144 ff.

${ }^{31}$ Woodford 1992, 433.

${ }^{32}$ Boardman 1984 Nr. 37, 39.
} 
hinten $\mathrm{zu}$ sehen. Auch diese Szenen folgen keiner konventionellen Ikonographie. Aphrodite und Adonis sitzen gewöhnlich nebeneinander, gegebenenfalls wird Adonis stehend dargestellt, wenn er eben zur Jagd aufbricht. Lediglich auf einem auf die Mitte des 2. Jahrhunderts n. Chr. datierten antiochischen Mosaik und auf einer Silberplatte aus dem 6. Jahrhundert stehen sie nebeneinander mit einem kleinen Eros in der Mitte. ${ }^{33}$ In der Szene der 'Leda mit dem Schwan' ist die Königstochter üblicherweise frontal oder im Profil zu sehen und der Schwan nähert sich ihr von vorne. Die von hinten dargestellt Leda und der sich von hinten nähernde Schwan finden sich auf einem Mosaik in Nicosia sowie auf einem um die Wende vom 4. zum 5. Jahrhundert entstandenen koptischen Relief. ${ }^{34}$

Im Zentrum der Platte sieht man Meleager auf dem Fell des Ebers sitzend und umgeben von seinen Jagdgefährten: an den Rändern je ein Dioskur, in der Mitte hinten Ankaios mit einem Doppelschwert in der Hand, neben ihm Theseus mit seiner Keule und unmittelbar hinter Meleager Atalante (Abb. 9) ${ }^{35}$ Ein solches Gruppenbild der Teilnehmer an der erfolgreichen Wildschweinjagd ist einzigartig unter den erhalten gebliebenen Darstellungen der Szene, auf denen die Anwesenheit der Onkel mütterlicherseits auf den tragischen Ausgang verweist.

Wenn wir nach alldem das Motiv suchen, das allen Szenen gemeinsam ist und sie zusammenhält, so ist dies ohne Zweifel das Motiv der Macht der Liebe, im Speziellen das beherrschende Motiv von Aphrodite und Eros. ${ }^{36}$ Diese Interpretation wird von der ersten Geschichte begründet, dem Urteil des Paris, auf Grund dessen Aphrodite den goldenen Apfel erhält. Mit dem Versprechen der Liebe der schönsten Frau siegt Aphrodite über die die Vernunft verkörpernde Athene und die Macht versprechende Hera. Im Hintergrund erscheint die die versprochene Liebe verkörpernde Helena. Im Perseus-Zyklus werden zwei Liebesgeschichten verdichtet: Zeus' unaufhaltsame Liebe zu Danaë und Perseus' Liebe zu Andromeda. In der Geschichte von Hippolytos und Phaidra können wir ein Beispiel für die einseitige und unerfüllte Liebe sehen. Hippolytos entscheidet sich anstelle der Liebe für die Jagd, was tödlich für ihn endet. Das Geschenk der Liebe sollte also nicht zurückgewiesen werden. Die Liebe von Pyramos und Thisbe erfüllt sich nicht, obwohl ihre Gefühle wechselseitig sind, das Schicksal aber spielt ihnen übel mit und sie sterben vor der Erfüllung bzw. verwandeln sich in Wassergötter. Erst danach sind sie, gleichsam über den Willen des Schicksals siegend, vereint. In der fünften Szene ist sicherlich die

\footnotetext{
${ }^{33}$ Servais-Soyez 1981 Nr. 31, 26.

${ }^{34}$ Kahil - Linant de Bellefonds 1992 Nr. 42, 52.

${ }^{35}$ Mango - Bennett 1994, 122-124.

${ }^{36}$ Mango - Bennett 1994, 147.
} 
Liebe von Paris und Helena zu sehen, aber mit der Anwesenheit sowohl des Löwen als auch der Dioskuren wird zugleich das Jagdmotiv betont. Im sechsten Segment werden drei Liebesgeschichten dargestellt, von denen zwei mit den Göttern, mit Zeus und Aphrodite, verbunden sind und eine mit dem Heros Meleager. In den letzteren beiden Geschichten erhält wieder die Jagd eine betonte Rolle: Meleager hält das Fell des erlegten Ebers, neben Adonis befindet sich ein Jagdhund und beide halten eine Lanze in der Hand. Im Zentrum der Platte, also in betonter Position, wird der Triumph der Liebe und der Jagd dargestellt: das Liebespaar Meleager und Atalante mit dem Fell des erlegten Ebers, um sie herum die Gruppe der Gehilfen. Keinerlei Anspielung gibt es dagegen auf den tragischen Ausgang der Geschichte, der einen Schatten auf den Triumph werfen würde.

Auf den Darstellungen sind also insgesamt acht Liebesgeschichten zu unterscheiden und in vier Fällen spielt auch die Jagd eine wichtige Rolle. Unter den vier Jagden enden drei später tragisch, davon findet sich aber in den Szenen keine Spur, vielmehr kommt die starke Liebe zum Ausdruck, auch dann, wenn sie nicht erwidert wird wie im Fall von Phaidra. Die Jagd ist sicherlich eine Weise der Ausübung der virtus, was die Anziehungskraft des erwählten Mannes noch steigert. Es ist kein Zufall, dass die Darstellung von Jagdszenen auch auf den kaiserzeitlichen Sarkophagen so beliebt war. Sie dienten dem Lobpreis der virtus des Verstorbenen. ${ }^{37}$ Die Jagd spielte nicht nur im übertragenen Sinn eine wichtige Rolle im Leben der zeitgenössischen Aristokratie. Dies zeigt gut das hervorragende Stück des Seuso-Schatzes, die große Jagdplatte, auf der im Zentrum oben Hirsch- und unten Wildschwein-, am Rand Bär-, Hirsch-, Leopard-, Onager-, Wildschwein-, Löwe-, Gazelle- und Haase-Jagdszenen zu sehen sind. ${ }^{38}$ Diese haben sich sicherlich auf dem Gut des Besitzers des Tafelsilbers zugetragen.

Das Liebesmotiv als ikonographisches Ordnungsprinzip ist auch in anderen Epochen der römischen kaiserzeitlichen Kunst häufig zu finden. Drei Szenen zieren die Wände des cubiculum des Hauses des Jason in Pompeji: Paris und Helena, Medea mit ihren zwei Kindern und Phaidra mit der Amme. ${ }^{39}$ Auf allen drei Bildern herrscht eine melancholische Stimmung, die auf den tragischen Ausgang der Liebesgeschichte verweist. Der Gesichtspunkt der Auswahl dürfte in diesem Fall die Warnung vor den Gefahren blinder Liebe gewesen sein. Auch im Fall der römischen kaiserzeitlichen Sarkophage ist die Liebe ein dominantes Thema, was von der Häufigkeit der Darstellungen von Aphrodite und

\footnotetext{
${ }^{37}$ Andreae 1980; Andreae 1985.

${ }^{38}$ Mango - Bennett 1994, 81-97.

${ }^{39}$ Bragantini - Sampaolo 2009, 240 f.
} 
Adonis, Selene und Endymion, Hippolytos und Phaidra, Theseus und Ariadne sowie Admetos und Alkestis unterstrichen wird. ${ }^{40}$ All diese Darstellungen sind als Ausdruck der unendlichen Liebe zu den Verstorbenen zu interpretieren, ohne dass diese Geschichten negative Konnotationen hätten. ${ }^{41}$ Aus den Parallelen zu den einzelnen Szenen geht hervor, dass die Liebe auch ein beliebtes Thema der kaiserzeitlichen Mosaike war. Geschichten des über alle und sogar über die Götter Macht ausübenden Eros erscheinen auch noch auf den Wandgemälden und Mosaiken eines öffentlichen Gebäudes im Gaza des 6. Jahrhunderts. ${ }^{42}$ Der christliche Rhetor Prokopios gibt eine detaillierte Beschreibung dieser Bilder. In seiner Einleitung erwähnt er Zeus' Beziehungen mit sterblichen Königstöchtern, unter ihnen Semele, Europa und Danaë. Eros verschonte mit seiner Erweckung von Liebesgefühlen auch seine eigene Mutter nicht, die sich so in Adonis verliebte. Aber auch Phaidra verschonte er nicht vor seinem Pfeil. Unter den dargestellten Themen finden wir diesen Gedanken auch in den Geschichten von Theseus und Ariadne sowie von Paris und Helena.

Auf Grund der erwähnten Parallelen ist festzustellen, dass die Darstellung von Liebesgeschichten durchgehend beliebt war, angefangen von der frühen Kaiserzeit bis zur Spätantike, ja sogar bis in die frühbyzantinische Zeit. Des Weiteren kann festgestellt werden, dass zur Illustration der alles bezwingenden Macht der Liebe im Wesentlichen die gleichen Geschichten aus der griechischrömischen Mythologie verwendet wurden. Was aber ist die Erklärung für diese Einheitlichkeit? Das Fortleben der mythologischen Bilder ist der antiken rhetorischen Bildung zu danken, welche auf den exempla der klassischen Autoren und deren Bildwelt aufgebaut war, was von großem Einfluss auf die Kultur der führenden Elite der Spätantike war. Die Haupthelden der Mythen waren exempla und Allegorien menschlicher Situationen und Verhaltensweisen. Auf diese Weise fanden die Götterbilder und Mythen ihren Platz bei Heiden und Christen gleichermaßen. ${ }^{43}$ Die Elemente der paideia standardisierten sich in der spätrömischen Zeit, blieben unverändert und bewahrten ihren Wert und ihre Bedeutung für die zeitgenössische Elite, von deren Identität sie einen wichtigen Teil bildeten.$^{44}$ Die Bewahrung der Tradition war ein Zeichen von Stabilität in einer sich ständig wandelnden Welt. Im Fall der Meleager-Platte ermöglichte die paideia das Erkennen und das Verständnis der Verbindung zwischen den verschiedenen mythologischen Szenen. Die Platten boten den Betrachtern die Möglichkeit, ihre literarischen Kenntnisse anzuwenden, die einen wichtigen

\footnotetext{
${ }^{40}$ Sichtermann - Koch 1975, s. v.

${ }^{41}$ Zanker - Ewald 2004, passim.

${ }^{42}$ Friedländer 1939 § 1, p. 24; Cameron 2011, 704.

${ }^{43}$ Bergmann 1999, 68.

${ }^{44}$ Leader - Newby 2004, 124.
} 
Bestandteil der paideia bildeten. Welchen wichtigen Stellenwert dabei die visuelle Kultur hatte, das kann auch mit Hilfe von Philostratos' Imagines festgestellt werden. ${ }^{45}$ Der Betonung der Bedeutung der paideia begegnen wir auch auf einem Sarkophag aus dem 3. Jahrhundert n. Chr.: Das verstorbene Kind sitzt als Philosophielehrer im Mittelpunkt und wird von seinen Zuhörern als Kinder-Musen umgeben. ${ }^{46}$

Nicht nur Prokopios' ekphrasis beweist, dass sich die Christen vor der heidnischen Mythologie nicht verschlossen, sondern auch Nonnos' Anfang des 5. Jahrhunderts geschriebene Dionysiaca. Dieses Werk enthält in 48 Büchern alles, was man von dieser Gottheit wusste, ohne dass irgendetwas darauf verweisen würde, dass Nonnos an die göttliche Existenz von Dionysos geglaubt hätte. Dionysos erscheint nicht als Erlöser, sondern einfach als Verkörperung des Weingenusses ${ }^{47}$ Klassische mythologische Szenen finden sich auf Silberobjekten bis zum 7. Jahrhundert. Es ist unvorstellbar, dass sie alle von Heiden stammen sollten. ${ }^{48}$ Offensichtlich hielt also auch die christliche Elite, zu der nach dem Christus-Monogramm auf der großen Jagdplatte (Abb. 10) zu schließen auch der Besitzer des Seuso-Schatzes gehörte, die heidnischen Themen zum Ausdruck der eigenen Identität für geeignet.

Es ist nicht mit Sicherheit festzustellen, in welchem Teil des Römischen Reiches der Besitzer des Seuso-Schatzes gelebt hat. In Verbindung mit den mit mythologischen Szenen verzierten Gefäßen hat sich die Meinung herauskristallisiert, dass sie aus östlichen Werkstätten vom Ende des 4. oder vom Anfang des 5. Jahrhunderts stammen. ${ }^{49}$ Ikonographische Untersuchungen insbesondere der Geschichte von Pyramos und Thisbe sprechen ebenfalls dafür, dass die Darstellungen östliche und spätantike Parallelen haben. Als charakteristischer Zug des Produzenten der Szenen kann festgestellt werden, dass er zur Bestimmung des Schauplatzes gerne personifizierte Gestalten als Nebenfiguren einsetzte, so etwa die Insel Seriphos, die Tyche Athens, den auf einer Syrinx spielenden Hirten und den Flussgott Eurotas. Diese Gestalten sind auch deshalb auffallend, weil sie nicht zur konventionellen Ikonographie der Darstellungen der einzelnen Geschichten gehören.

Zusammenfassend kann also festgestellt werden, dass der rote Faden der dargestellten Geschichten die alles bezwingende Liebe ist, das wichtigste Gefühl, welches das menschliche Leben antreibt. So wie Ovid in seinen Metamorphosen bei der Beschreibung der Geschichte von Pyramos und Thisbe immer

\footnotetext{
${ }^{45}$ Ghedini-Colpo-Novello 2004.

${ }^{46}$ Zanker - Ewald 2004, 216.

${ }^{47}$ Cameron 2011, $700 \mathrm{f}$.

${ }^{48}$ Cameron 2011, $705 \mathrm{f}$.

${ }^{49}$ Visy 2012, passim; Mango - Bennett 1994, 152.
} 
wieder die Wörter amor und amans verwendet, so kehrt auch hier in den einzelnen Szenen dieses Motiv immer wieder. Der Nebenstrang der Geschichten ist die Jagd, die sowohl im wörtlichen als auch im übertragenen Sinn verstanden werden kann. Es handelt sich um eine aristokratische Vergnügung und zugleich auch Praxis der Tugend, an der auch Frauen teilnehmen konnten, wie die Szene von Helena und Atalante zeigt. Eine noch engere Bestimmung und Interpretation des ikonographischen Programms hielten wir für an den Haaren herbeigezogen und für zu riskant. Auch wenn wir zu Erklärung des Vorkommens des Liebesmotivs nicht unbedingt einen aktuellen Anlass suchen müssen, so ist doch der Gedanke verlockend, die zeitgenössische Funktion des Schatzes als Hochzeitsgeschenk zu bestimmen. Wir neigen also zur Erklärung, dass Seuso, der wohlhabende Besitzer des Schatzes, dieses einzigartige Tafelsilber Anfang des 5. Jahrhunderts als Hochzeitsgeschenk erhalten hat. Der auf die Jagdplatte gravierte Wunsch hat sich zu unserem Glück erfüllt: H(a)ec vascula durent per saecula multa (Mögen diese Gefäße noch über viele Jahrhunderte dauern).

\section{Bibliographie}

Andreae $1980=$ Andreae, B.: Die römischen Jagdsarkophage. Berlin.

— 1985 = Andreae, B.: Die Symbolik der Löwenjagd . Opladen.

Bergmann 1999 = Bergmann, M.: Chiragan, Aphrodisias, Konstantinopel. Zur mythologischen Skulptur der Spätantike. Wiesbaden.

Bragantini - Sampaolo 2009 = Bragantini, I. - Sampaolo,V.: La pittura pompeiana Napoli.

Boardman 1984 = Boarman, J.: Atalante. LIMC II. 940-950.

Cameron 2011 = Cameron, A.: The Last Pagans of Rome. Oxford

Friedländer 1939 = Friedländer, P.: Spätantiker Gemäldezyklus in Gaza des Prokopios von Gaza. Citta del Vaticano.

Gesztelyi $2015=$ Gesztelyi, T.: Phaedras Brief an Hippolytus. Ovids Brief (Her. 4) in der römischen bildenden Kunst. ACD 51, 59-75.

Ghedini - Colpo - Novello 2004 = Ghedini, F. - Colpo, I. - Novello, M.: Le Immagini di Filostrato Minore. Roma.

Kahil - Linant de Bellefonds1992 = Kahil, L. - Linant de Bellefonds, P.: Leda. LIMC VI. 231246.

Knox 1989 = Knox, P. E.: Pyramus and Thisbe in Cyprus. Harvard Studies 92, 315-328.

Kossatz-Deissmann 1994 = Kossatz-Deissmann, A.: Paridis iudicium. LIMC VII. 176-188.

Leader-Newby 2004 = Leader-Newby, R. E.: Silver and Society in Late Antiquity: Functions and Meanings of Silver Plate in the Fourth to Seventh Centuries. Ashgate.

Linant de Bellefonds 1990 = Linant de Bellefonds, P.: Hippolytos I. LIMC V. 445-464.

— 1994 = Linant de Bellefonds P.: Pyramos et Thisbe. LIMC VII. 605-607.

— 1994a = Linant de Bellefonds, P.: Pyrame et Thisbé: l'autre légende. In: Tranquillitas. Mélanges en l'honneur de Tran tam Tinh. 343-352. 
Maffre 1981 = Maffre, J. J.: Akrisios. LIMC I. 449-452.

— 1986 = Maffre, J. J.: Danae. LIMC III. 325-337.

Mango - Bennett 1994 = Mango, M. M.- Bennett, A.:The Seuso Treasure. Ann Arbor.

Roccos 1994 = Roccos, L. J.: Perseus. LIMC VII. 332-348.

Schauenburg 1981 = Schauenburg, K.: Andromeda I. LIMC I. 774-790.

Servais-Soyez 1981 = Servais-Soyez, B.: Adonis. LIMC I. 222-229.

Sichtermann - Koch 1975 = Sichtermann, H. - Koch, G.: Griechische Mythen auf römischen Sarkophagen. Tübingen.

Türk 1937 = Türk, G.: Thisbe. RE VI A. 286-287.

Visy 2012 = Visy, Zs. (ed.): A Seuso-kincs és Pannonia. The Seuso Treasure and Pannonia. Pécs.

Woodford 1992 = Woodford, S.: Meleagros. LIMC VI. 414-435.

Zanker - Ewald 2004 = Zanker, P. - Ewald, B. Chr.: Mit Mythen leben. Die Bilderwelt der römischen Sarkophage. München.

\author{
Abbildungen: \\ 1. Iudicium Paridis (Mango-Bennett) \\ 2. Perseus-Zyklus (Mango-Bennett) \\ 3. Hippolytos und Phaidra (Mango-Bennett) \\ 4. Hippolytos und Phaidra auf den zwei Eimer und auf einer Kanne (Mango-Bennett) \\ 5. Pyramos und Thisbe (Mango-Bennett) \\ 6. Pyramos und Thisbe auf einem Mozaik in Nea Paphos (Knox) \\ 7. Helene, Paris, Dioskuroi (Mango-Bennett) \\ 8. Leda, Atalante, Aphrodite (Mango-Bennett) \\ 9. Meleager und seine Gefährte (Mango-Bennett) \\ 10. Mitte des grossen Jagdtellers (Mango-Bennett)
}

(ISSN $0418-453 X)$ 